\title{
Kaempferol promotes bone formation in part via the mTOR signaling pathway
}

\author{
JING ZHAO ${ }^{1-4}$, JUE WU $^{5}$, BINWU XU ${ }^{6}$, ZHEN YUAN $^{2-4}$, YU LENG $^{7}$, \\ $\mathrm{JUN}_{\mathrm{MIN}}{ }^{8}$, XIAOYONG LAN ${ }^{9}$ and $\mathrm{JUN}^{2} \mathrm{LUO}^{2-4}$ \\ ${ }^{1}$ Department of Neurology, The Second Affiliated Hospital of Nanchang University; \\ ${ }^{2}$ Regeneration and Rehabilitation Engineering Research Institute on Bone and Nerve of Jiangxi; \\ ${ }^{3}$ Orthopedics Research Institute of Jiangxi, Nanchang University; ${ }^{4}$ Department of Rehabilitation, \\ The Second Affiliated Hospital of Nanchang University, Nanchang, Jiangxi 330000; \\ ${ }^{5}$ Laboratory of Translational Medicine, Chinese PLA General Hospital, Beijing 100853; ${ }^{6}$ Department of Orthopedics, \\ The Second Affiliated Hospital of Nanchang University, Nanchang, Jiangxi 330000; ${ }^{7}$ Department of Emergency, \\ The First People's Hospital of Jiujiang City, Jiujiang, Jiangxi 332000; Departments of ${ }^{8}$ Rehabilitation and ${ }^{9}$ Orthopedics, \\ The Third Affiliated Hospital of Nanchang University, Nanchang, Jiangxi 330008, P.R. China
}

Received November 2, 2018; Accepted July 9, 2019

DOI: $10.3892 / \mathrm{mmr} .2019 .10747$

\begin{abstract}
Previous research indicates that kaempferol (Kae) promotes osteogenesis, but its underlying mechanism of action remains unclear. The present study hypothesized that the osteogenic effects of Kae were mediated through mammalian target of rapamycin (mTOR). To validate this hypothesis, bone marrow mesenchymal stem cells (BMSCs) from ovariectomized (OVX) rats were differentiated into osteoblasts. The bone mineral density and bone microarchitecture of the OVX rats was measured in vivo, while osteogenesis was evaluated in vitro via Alizarin Red S staining and alkaline phosphatase activity measurements in cultured BMSCs. The levels of phosphorylated eukaryotic translation initiation factor 4E-binding protein 1 ( $\mathrm{p}-4 \mathrm{E} / \mathrm{BP} 1)$ and phosphorylated ribosomal protein S6 kinase B1 (p-S6K), and the expression of Runt-related transcription factor 2 and Osterix, were concurrently quantified by western blot analysis. The data suggested that Kae prevented OVX-induced osteoporosis in rats by promoting osteoblastogenesis. Furthermore, treatment with Kae in rat BMSCs enhanced mineralization, elevated ALP activity, increased the expression levels of Runx-2 and Osterix and increased the levels of $\mathrm{p}-\mathrm{S} 6 \mathrm{~K}$ and decreased the levels of $\mathrm{p}-4 \mathrm{E} / \mathrm{BP} 1$ and,
\end{abstract}

Correspondence to: Professor Jun Luo, Department of Rehabilitation, The Second Affiliated Hospital of Nanchang University, 1 Minde Road, Nanchang, Jiangxi 330000, P.R. China E-mail: luojun1786@163.com

Abbreviations: Kae, kaempferol; BMSCs, bone mesenchymal stem cells; mTOR, mammalian target of rapamycin; OVX, ovariectomized; Rapa, rapamycin

Key words: Kae, osteoporosis, BMSCs, mTOR, Rapa consistent with its ability to promote osteoblast differentiation. In contrast, treatment with rapamycin, an mTOR inhibitor, produced the opposite phenotype. Taken together, these data suggested that the protective effects of Kae in BMSCs and in the OVX rat model resulted from the induction of osteogenesis via mTOR signaling, or at least partially via the regulation of downstream effectors of the mTOR pathway.

\section{Introduction}

Osteoporosis is described as a 'silent disease', characterized by gradual bone loss that occurs in the absence of other symptoms over a period of years $(1,2)$. Due to its increasing prevalence, osteoporosis severely affects human health and quality of life (1). Osteoporosis is a metabolic disease that is common in postmenopausal women and the elderly (1). Phenotypically, it is characterized by bone loss, bone microstructural damage, bone fragility and increased susceptibility to fractures (1). The approved medications for the prevention and treatment of osteoporosis in clinical practice are frequently ineffective and cause adverse reactions (1-5). New therapeutic strategies are therefore urgently required.

Kaempferol (Kae; Fig. 1) is a type of natural flavonoid, extracted from the rhizome of Kaempferia galangal L. (6). As a natural flavonol, Kae is present in a variety of Chinese medicinal herbs, plants, fruits and beans (6), and is prized for its medicinal properties, which include anti-inflammatory (7) and antitumoral (8) effects, as well as being beneficial for the treatment of diabetes (9), cardiovascular disease (10) and osteoporosis (11). Kae has been previously confirmed to be beneficial to bone microarchitecture by increasing bone density and reversing osteoporosis in ovariectomized (OVX) rats. However, the precise mechanism(s) governing these effects have not been defined.

Mammalian target of rapamycin (mTOR) is a member of the phosphatidylinositol 3-kinase-related kinase family 
of protein kinases (12). mTOR functions through two structurally and functionally distinct multi-protein complexes, mTORC1 and mTORC2, which are involved in cell growth, proliferation, survival, protein synthesis, autophagy and transcription (13). mTOR is also an important regulator of bone metabolism known to promote osteoblastic differentiation and increase bone matrix synthesis $(14,15)$. In addition, mTORC1 and $\mathrm{mTORC} 2$ have been implicated in the regulation of bone homeostasis (16-18).

Therefore, mTOR was hypothesized to be a novel target for the development of new and effective osteoporosis therapies. The aim of the present study was to investigate whether Kae was able to enhance the osteogenic differentiation and function of bone marrow mesenchymal stem cells (BMSCs) via mTOR activation.

\section{Materials and methods}

Reagents. Kae (purity $>98 \%$ ) was purchased from the National Institute for the Control of Pharmaceutical and Biological Products (Beijing, China). Kae was dissolved in DMSO and diluted to $0.01 \%$ in PBS. Rapamycin (Rapa), a specific inhibitor of mTOR, was purchased from Selleck Chemicals. The Alizarin Red S (ARS) staining buffer and alkaline phosphatase (ALP) detection kits were purchased from Nanjing Jiancheng Bioengineering Institute. Anti-runt-related transcription factor 2 (Runx2; cat. no. ab23981) and anti-Osterix (cat. no. ab22552) was purchased from Abcam. Anti-eukaryotic translation initiation factor 4E-binding protein 1 (4E/BP1; cat. no. 94525), anti-phosphorylated (p)-4E/BP 1 (cat. no. 2855) and anti-ribosomal protein S6 kinase B1 (S6K1; cat. no. 9202), anti-p-S6K1 (cat. no. 9204) was obtained from Cell Signaling Technologies, Inc. Horseradish peroxidase-labeled anti-immunoglobulin $\mathrm{G}$ secondary antibody (goat anti-mouse lgG; cat. no. SA00001-1; and goat anti-rabbit lgG; cat. no. SA00001-2) was obtained from ProteinTech Group, Inc. Anti- $\beta$-actin antibody (cat. no. KL002) was provided by Nanjing Jiancheng Bioengineering Institute.

Animals. A total of 30 adult (age, 6-8 weeks) female Sprague-Dawley (SD) rats weighing 180-220 g were obtained from the Nanchang University Laboratory Animal Center (Nanchang, China) and maintained under a 12-h dark/light cycle at $22-25^{\circ} \mathrm{C}$ and $40-70 \%$ humidity. Animals were allowed access to food and water ad libitum. Experiments were performed according to the Guide for the Care and Use of Laboratory (National Institutes of Health), and were approved by the Ethics Committee of Nanchang University (no. 2017-0122).

Experimental groups and treatments. A total of 30 rats were randomly divided into five groups ( $\mathrm{n}=6$ in each group) as follows: i) Sham group, in which the abdominal cavities of the rats were opened and fat tissue around the ovaries removed; ii) OVX group, in which the rats were ovariectomized; iii) Kae treatment group (OVX + Kae), in which the OVX rats were continuously given Kae $(100 \mathrm{mg} / \mathrm{kg} / \mathrm{day})$ via gavage for 8 weeks; iv) Kae and Rapa administration group (OVX + Kae + Rapa), in which the rats were treated as in the Kae treatment group and in addition received intraperitoneal injections of Rapa ( $0.2 \mathrm{mg} / \mathrm{kg} /$ day) for 8 weeks; and v) Rapa group (OVX + Rapa), in which the OVX rats received intraperitoneal injections of Rapa $(0.2 \mathrm{mg} / \mathrm{kg} / \mathrm{day})$ for 8 weeks. The dose and timing of Kae and Rapa administration were ascertained in preliminary experiments. Briefly, the OVX rats were given Kae at 25,50 and $100 \mathrm{mg} / \mathrm{kg} / \mathrm{day}$. Bone mineral density (BMD) was detected after 8 weeks to select the working concentration. Kae at $100 \mathrm{mg} / \mathrm{kg} /$ day was the most effective concentration in improving BMD in OVX rats. Rapa was administered at $0.2 \mathrm{mg} / \mathrm{kg} / \mathrm{day}$ according to the manufacturer's protocol (Selleck Chemicals). Subsequent experiments were performed following the 8 weeks of treatment.

Assessment of BMD and bone microarchitecture. Following treatment, the rats were sacrificed and their right femurs and tibias were dissected. The $2 \mathrm{D}$ total bone mineral content was used to calculate the BMD as previously described (19). The right femurs of the rats were analyzed using dual-energy X-ray absorptiometry with a Lunar Prodigy Advance system (version 13.6; GE Healthcare).

Based on the median values of total BMD, selected trabecular microarchitecture of the femoral metaphysis was evaluated using micro-computed tomography (micro-CT; Scanco Medical AG) with Scanco image processing language software (version 5.08b; Scanco Medical AG). Scans were performed from the proximal growth plate in the distal direction $(18 \mu \mathrm{m} / \mathrm{slice})$ as the distal femur has a high concentration of trabecular bone compared with the proximal and middle regions of the femur. A volume of interest (VOI) was selected, defined as the cross-sectional area spanning 100 slices from the proximal growth plate. The $2 \mathrm{D}$ scans were used to produce 3D reconstructions of the bone microarchitecture, which were used to measure bone morphometric parameters of the selected VOI, including the bone volume fraction [bone volume/tissue volume (BV/TV)], trabecular number (Tb.N), trabecular separation (Tb.Sp), trabecular thickness (Tb.Th) and structure model index (SMI). The operator conducting the CT analysis was blinded to the treatments associated with the specimens. All examinations were conducted according to the principles and procedures described in the most recent National Research Council publication of the Guide for the Care and Use of Laboratory Animals and the ARRIVE guidelines (20).

Isolation of rat BMSCs. BMSCs were flushed from the femurs and tibias of normal SD and OVX rats with PBS in a biosafety cabinet, using a $5 \mathrm{ml}$ syringe fitted with a needle (21G). Mononucleated cells were isolated by density gradient centrifugation at $400 \mathrm{x} \mathrm{g}$ for $20 \mathrm{~min}$ at room temperature in rat lymphocyte separation medium (TBD Science) at a concentration of $1.091 \mathrm{~g} / \mathrm{ml}$. Isolated cells were cultured in low-glucose DMEM (HyClone; GE Healthcare Life Sciences) containing $15 \%$ fetal bovine serum (HyClone; GE Healthcare Life Sciences). All cells were maintained in a $37^{\circ} \mathrm{C}$ incubator within an atmosphere containing $5 \% \mathrm{CO}_{2}$. BMSCs were identified by CD44 and CD34 labeling (21). The culture medium was regularly replaced and cells were passaged after reaching $70-80 \%$ confluence. Third-generation BMSCs were harvested for subsequent experiments.

Cytotoxicity assays. The cytotoxicity of BMSCs was determined using an MTT assay. Briefly, rat BMSCs were plated 


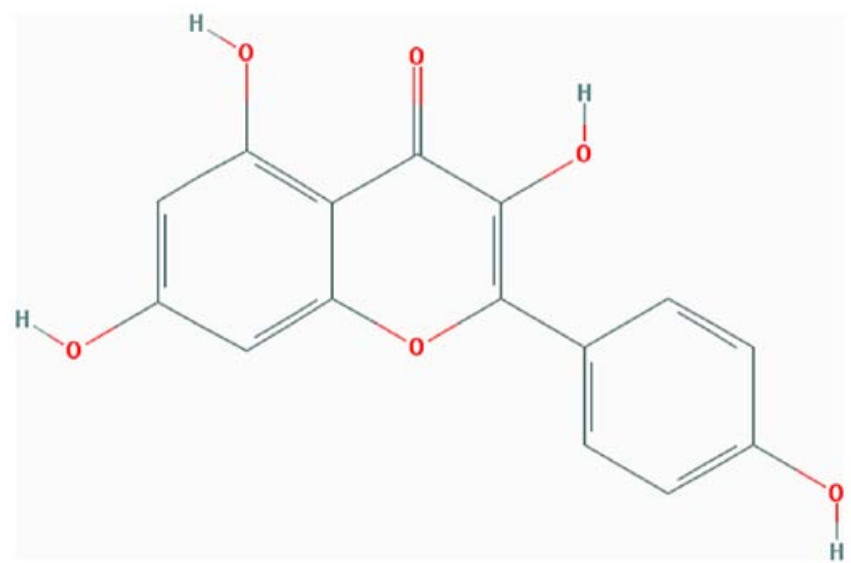

Figure 1. Chemical structure of kaempferol (Pubchem CID: 5280863).

into 96-well plates at a density of $1 \times 10^{5}$ cells/well. After $24 \mathrm{~h}$ of culture in a $5 \% \mathrm{CO}_{2}$ incubator at $37^{\circ} \mathrm{C}$, adherent cells were treated with a range of Kae concentrations $(0.1,1,10$ and $100 \mu \mathrm{M})$ for $24 \mathrm{~h}$. The MTT reagent $(10 \mu \mathrm{l} ; 10 \mathrm{mg} / \mathrm{ml})$ was subsequently added to each well, and cells were incubated for a further 4- $6 \mathrm{~h}$ in a $5 \% \mathrm{CO}_{2}$ incubator at $37^{\circ} \mathrm{C}$. The medium was removed and $100 \mu \mathrm{l}$ DMSO was added to each well. Plates were shaken for $10 \mathrm{~min}$, and the absorbance was measured at $492 \mathrm{~nm}$ using a Spectra Max Paradigm microplatereader (Molecular Devices, LLC). Cytotoxicity was calculated as follows: Cytotoxicity $(\%)=(1-a b s o r b a n c e$ of sample/absorbance of control $) \times 100$.

Experimental groups and treatments. Third-passage BMSCs were randomly divided into 5 groups: i) Control group, in which BMSCs derived from normal rats were treated with osteogenic induction medium (Cyagen Biosciences, Inc.) to induce osteoblast differentiation; ii) OVX group, in which BMSCs derived from OVX rats were treated in the same way as the control group; iii) OVX + Kae group, in which BMSCs derived from OVX rats were incubated with $0.1,1,10$ or $100 \mu \mathrm{M}$ Kae and treated in the same way as the control group; iv) OVX + Kae + Rapa group, in which BMSCs derived from OVX rats were incubated with Kae and $10 \mu \mathrm{M}$ Rapa and then treated in the same way as the control group; v) OVX + Rapa group, where BMSCs derived from OVX rats were incubated with $10 \mu \mathrm{M}$ Rapa and treated in the same way as the control group. The dose of Rapa was determined during preliminary experiments. Briefly, MTT was detected to evaluate the optimal concentration of Rapa. Rapa (at $0.1,1,10$ or $100 \mu \mathrm{M}$ ) was incubated with BMSCs. Rapa was found to exhibit a concentration-dependent cytotoxic effect. Therefore, $10 \mu \mathrm{M}$ was selected. BMSCs were harvested at day 15 in all groups.

ARS staining. ARS staining was used to assess the osteogenic differentiation of BMSCs. Briefly, rat BMSCs were plated into 24-well plates at a density of $2.5 \times 10^{5}$ cells/well. After induction of osteogenesis, cells were fixed with $95 \%$ alcohol for $15 \mathrm{~min}$ at room temperature and the BMSCs were stained with ARS for $5 \mathrm{~min}$ at room temperature. Mature osteoblasts that differentiated from rBMSCs displayed intense brown-red staining after 5 min of ARS staining (magnification, x40). Cells were imaged at $\mathrm{x} 400$ magnification by light microscopy, after the addition of $10 \%(\mathrm{w} / \mathrm{v})$ cetylpyridinium chloride to precipitate calcium ions. The absorbance of each well was measured at $562 \mathrm{~nm}$ using a Spectra Max Paradigm microplatereader (Molecular Devices, LLC).

Alkaline phosphatase activity assay. The effects of Kae on osteoblasts were assessed using an ALP assay kit. The absorbance/optical density (OD) of each sample was measured at $492 \mathrm{~nm}$ using a microplate reader. ALP activity in the osteogenic induction medium (Cyagen Biosciences, Inc.) was determined as follows: ALP activity (Jinshi unit $/ 100 \mathrm{ml})=(\mathrm{T}-\mathrm{B}) /(\mathrm{S}-\mathrm{B}) \times 0.02 \mathrm{mg} / \mathrm{ml} \times 100 \mathrm{ml} \mathrm{x}$ a, where $\mathrm{T}=\mathrm{OD}$ of the test sample; $\mathrm{B}=\mathrm{OD}$ of the blank; $\mathrm{S}=\mathrm{OD}$ of the standard; and a=dilution factor of the sample. According to the conditions above, a Jinshi unit is defined as the ALP activity that releases of $1 \mathrm{mg}$ of phenol every $15 \mathrm{~min}$ after mixing with $100 \mathrm{ml}$ liquid matrix at $37^{\circ} \mathrm{C}$.

Western blot analysis. Cells were lysed in RIPA lysis buffer containing $50 \mathrm{mM}$ Tris- $\mathrm{HCl}(\mathrm{pH} 7.4), 150 \mathrm{mM} \mathrm{NaCl}, 1 \%$ sodium deoxycholate, $1 \%$ NP-40, $1 \mathrm{mM}$ PMSF and $1 \mathrm{mM}$ EDTA. Then, extracts were centrifuged at $24,750 \mathrm{x}$ g at $4^{\circ} \mathrm{C}$ for $15 \mathrm{~min}$ to remove insoluble material. Total protein concentrations were determined using a bicinchoninic acid assay (Beyotime Institute of Biotechnology), according to the manufacturer's protocol. An equivalent quantity of total protein $(40 \mu \mathrm{g})$ per well was diluted in sample buffer containing $100 \mathrm{mM}$ dithiothreitol and heated to $98^{\circ} \mathrm{C}$ for $5 \mathrm{~min}$. Lysates were separated using 10-15\% SDS-PAGE (Bio-Rad Laboratories, Inc.) and subsequently transferred to PVDF membranes. The membranes were blocked in 5\% non-fat dry milk for $2 \mathrm{~h}$ at room temperature and incubated overnight at $4^{\circ} \mathrm{C}$ using primary antibodies (1:500) against $\beta$-actin, Runx2, 4E/BP 1 , p-4E/BP1, S6K1 and p-S6K1. Membranes were washed in TBS with Tween-20 (TBS-T), and incubated with horseradish peroxidase-conjugated secondary antibody $(1: 2,000)$ at room temperature for $1 \mathrm{~h}$. Membranes were washed three times for $20 \mathrm{~min}$ in TBS-T, and protein bands were visualized by enhanced chemiluminescence (Proteintech Group, Inc.). Band intensities were measured and quantitated using Quantity One software (version 4.6.6; Bio-Rad Laboratories, Inc.) and $\beta$-actin was used for normalization.

Statistical analysis. SPSS (version 20.0; IBM Corp.) was used for statistical analysis. Data are presented as the mean \pm SEM from six independent experiments. The variance homogeneity test and one-way ANOVA were performed between groups. Newman-Keuls test was used following ANOVA. $\mathrm{P}<0.05$ was considered to indicate a statistically significant difference.

\section{Results}

Kae ameliorates $O V X$-induced osteoporosis in rats. The 3D trabecular bone microarchitecture, used for the assessment of distal femoral metaphysis, was calculated from micro-CT images (Fig. 2). The mean BV/TV, Tb.N, Tb.Th and BMD values in the OVX group were significantly lower than those of the sham group, while Tb.Sp and SMI were higher $(\mathrm{P}<0.05)$. Compared with the OVX group, BV/TV, Tb.N, Tb.Th and BMD values were significantly greater, while Tb.Sp and SMI 
A

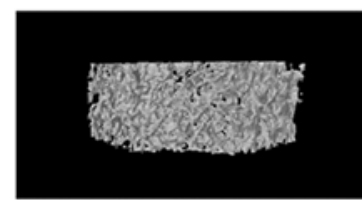

Sham

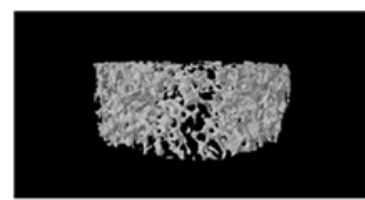

OVX

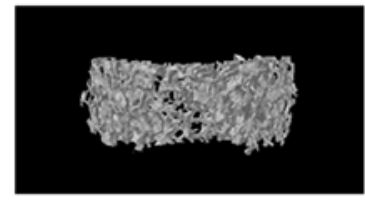

$\mathrm{OVX}+\mathrm{Kae}$

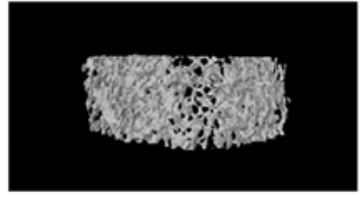

$\mathrm{OVX}+\mathrm{Kae}+\mathrm{Rapa}$

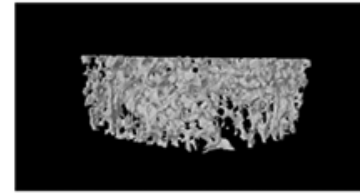

$\mathrm{OVX}+\mathrm{Rapa}$
B

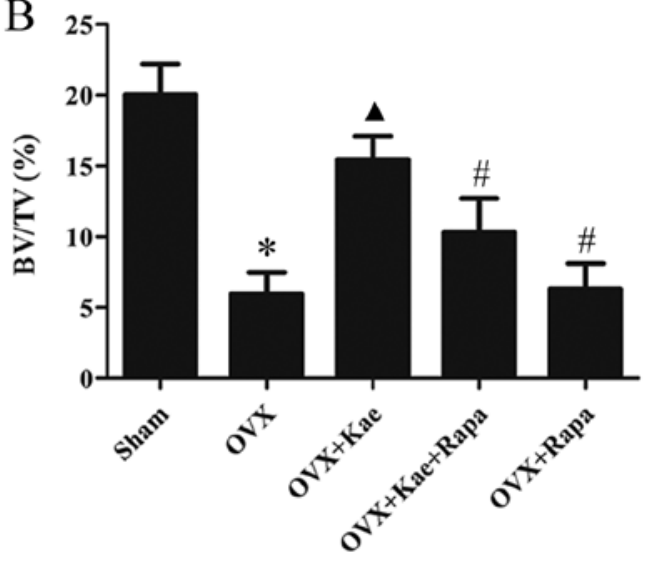

D

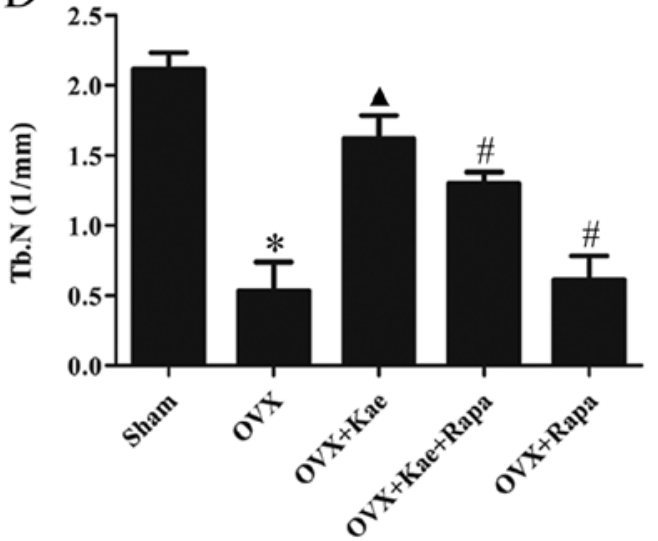

F

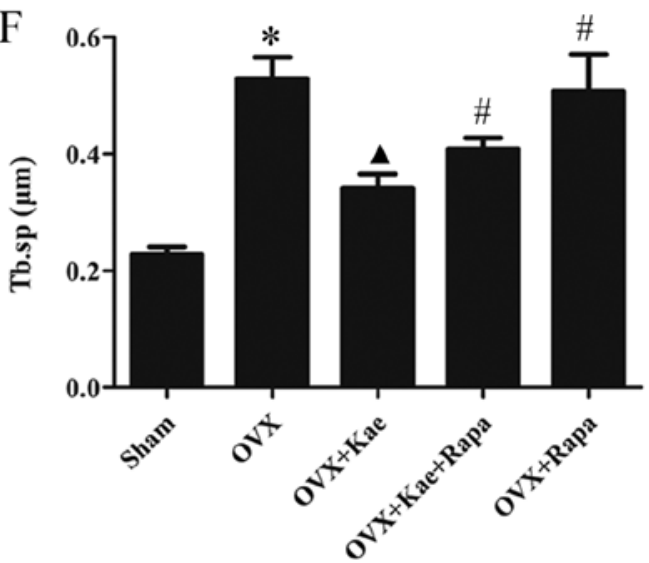

C

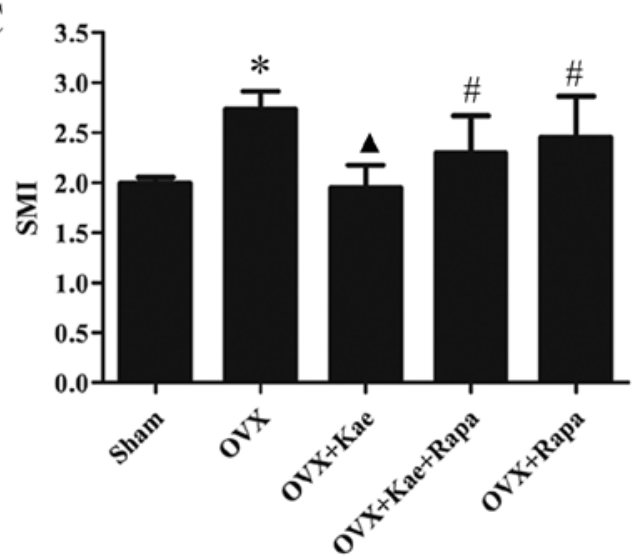

E
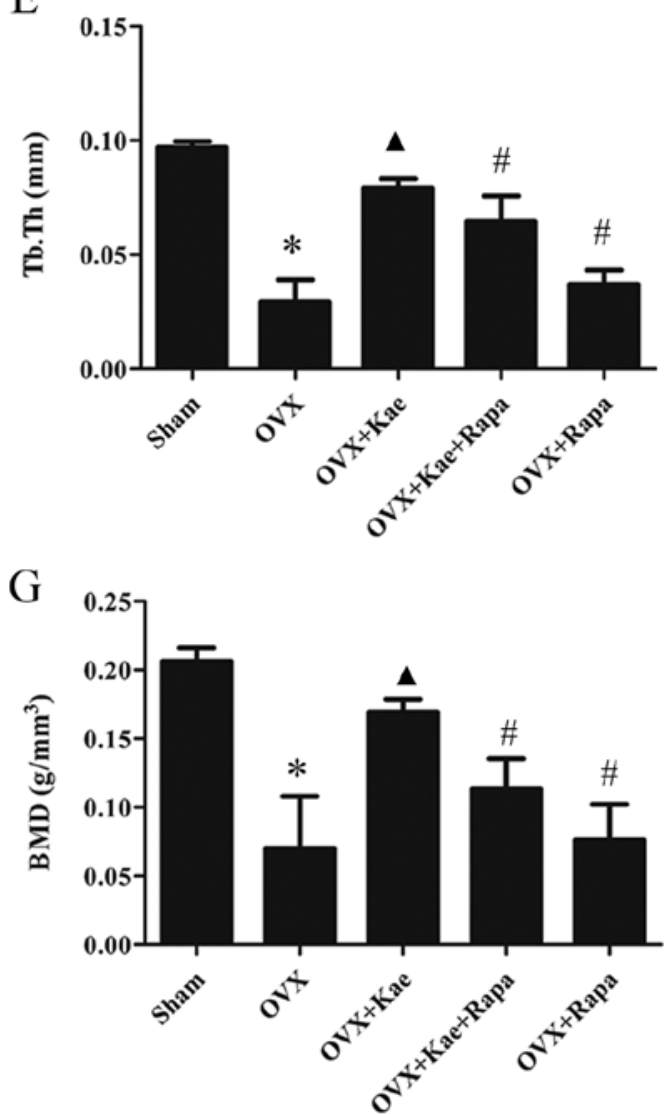

Figure 2. Effects of Kae on bone microarchitecture in rats. (A) Representative trabecular bone microarchitecture of the femoral metaphysis for each group, obtained from micro-CT images. Quantification of various parameters of bone microarchitecture for each group: (B) BV/TV, (C) SMI, (D) Tb.N, (E) Tb.Th, and (F) Tb.Sp. (G) BMD parameters measured using micro-CT in each group. The data are expressed as the mean $\pm \mathrm{SEM}$ (n=6). $\mathrm{P}<0.05 \mathrm{vs.}$ control group; $\Delta \mathrm{P}<0.05$ vs. OVX group; ${ }^{\#} \mathrm{P}<0.05$ vs. OVX + Kae group. Kae, kaempferol; micro-CT, micro-computed tomography; BV/TV, bone volume/tissue volume; Tb.N, trabecular number; Tb.Sp, trabecular separation, Tb.Th, trabecular thickness; SMI, structure model index; BMD, bone mineral density; Rapa, rapamycin; OVX, ovariectomized. 
were lower in the OVX + Kae group $(\mathrm{P}<0.05)$. Thus, treating OVX rats with Kae significantly improved bone microarchitecture and mass. The opposite trend was observed in the OVX + Kae + Rapa and OVX + Rapa groups: OVX rats treated with Kae + Rapa or Rapa only exhibited lower BV/TV, Tb.N and Tb.Th values and higher Tb.Sp and SMI values compared to the OVX + Kae group ( $\mathrm{P}<0.05$ vs. OVX + Kae) .

Assessment of the optimal Kae dose that promotes osteoblast differentiation. The cytotoxicity of various concentrations of Kae was tested on BMSCs. As shown in Fig. 3, Kae displayed a concentration-dependent cytotoxic effect, demonstrating high cytotoxicity at $100 \mu \mathrm{M}$. Additionally, it was observed that Kae induced osteoblast differentiation (Fig. 4). On day 15 following osteogenic induction, ARS staining revealed increased calcium deposition with increasing Kae concentrations. However, the number of osteoblasts unexpectedly decreased when cells were treated with $100 \mu \mathrm{M}$ Kae. From the range of concentrations tested, the optimal concentration of Kae that promoted osteoblast differentiation was $10 \mu \mathrm{M}$. This concentration was therefore used in subsequent experiments.

Kae promotes osteogenic differentiation. ARS staining was used to assess the induction of osteogenesis in BMSCs. As illustrated in Fig. 5, following the induction of osteogenic differentiation, the rate of calcific nodule formation decreased in the OVX group compared to the control group $(\mathrm{P}<0.05)$. Moreover, treatment with Kae significantly increased the number of calcified nodules in the OVX + Kae group $(\mathrm{P}<0.05$ vs. OVX group). However, the protective effect of Kae on osteoblasts was antagonized by the co-administration of Rapa $(\mathrm{P}<0.05$ vs. OVX $+\mathrm{Kae})$

Kae increases ALP expression in BMSCs. ALP activity was measured during osteogenesis. As shown in Fig. 6, the expression of ALP in the OVX group was significantly reduced compared with the control group $(\mathrm{P}<0.05)$. In addition, ALP activity was significantly higher in the OVX $+\mathrm{Kae}$ group compared to the OVX group ( $\mathrm{P}<0.05$ vs. OVX group). Pretreatment with Kae + Rapa or with Rapa alone led to reduced levels of ALP activity compared to Kae treatment alone $(\mathrm{P}<0.05)$.

Kae upregulates Runx2 and Osterix expression. Western blot analysis was used to assess the expression of the osteogenesis-related transcription factors Runx 2 and Osterix during osteogenesis. Fig. 7 demonstrates that a significant downregulation of Runx 2 and Osterix expression occurred in the OVX group compared with the control group $(\mathrm{P}<0.05)$. Kae significantly increased the expression of Runx 2 and Osterix compared with the OVX group $(\mathrm{P}<0.05)$. However, their expression was significantly lower in the OVX + Kae + Rapa and OVX + Rapa groups compared with the OVX + Kae group $(\mathrm{P}<0.05)$.

Kae alters the phosphorylation of $4 E / B P 1$ and S6K1. Western blot analysis was used to determine the levels of phosphorylated and total 4E/BP1 and S6K1, important downstream regulators of the mTOR pathway. As shown in Fig. 8, the levels of $\mathrm{p}-4 \mathrm{E} / \mathrm{BP} 1$ in the $\mathrm{OVX}+\mathrm{Kae}$ group were significantly

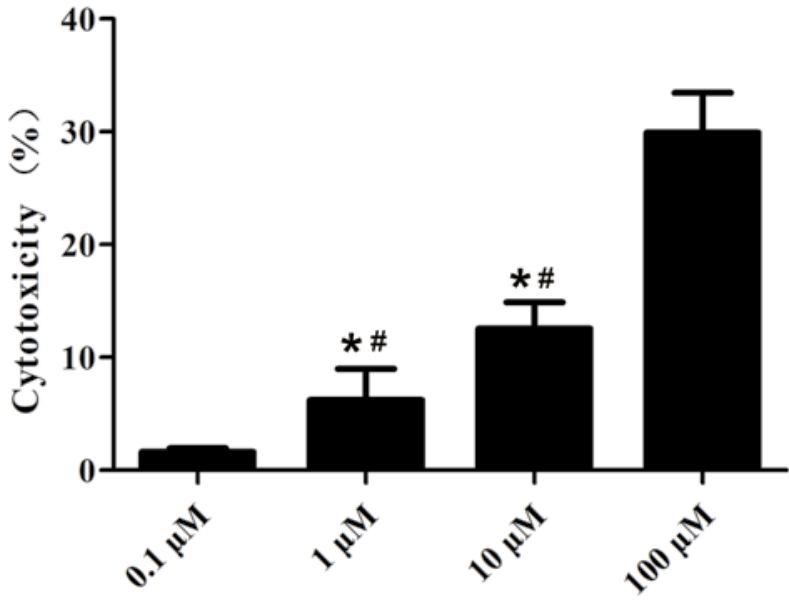

Figure 3. Effects of Kae on cell cytotoxicity. BMSCs were treated with different concentrations of Kae and cell cytotoxicity was assessed via an MTT assay. The data are expressed as the mean \pm SEM of six individual experiments. ${ }^{*} \mathrm{P}<0.05$ vs. $0.1 \mu \mathrm{M}$ and ${ }^{\#} \mathrm{P}<0.05$ vs. $100 \mu \mathrm{M}$. Kae, kaempferol; BMSCs, bone mesenchymal stem cells.

lower than in the OVX group $(\mathrm{P}<0.05)$. However, an notably increased level of $4 \mathrm{E} / \mathrm{BP} 1 \mathrm{p}-4 \mathrm{~EB} / \mathrm{P} 1$ was observed in the $\mathrm{OVX}+\mathrm{Kae}+\mathrm{Rapa}$ and OVX + Rapa groups. In addition, Kae increased S6K1 and p-S6K1 levels (Fig. 9; P<0.05 vs. OVX group). Conversely, the levels of S6K1 and p-S6K1 were significantly lower in the OVX + Kae + Rapa and OVX + Rapa groups compared with the OVX + Kae group $(\mathrm{P}<0.05)$. Taken together, these results indicated that Kae may promote osteogenesis through mTOR signaling as 4E/BP1 was activated by mTOR, whereas S6K1 exhibited the opposite trend.

\section{Discussion}

Estrogen has a significant role in bone metabolism (22), inhibiting bone resorption and stimulating bone formation (23). Flavonoids are estrogen-like chemicals used as estrogen substitutes, which may exert protective effects against postmenopausal bone loss $(11,24)$. In addition, Kae is a phytoestrogen that possesses osteogenic properties (6). Guo et al (25) demonstrated that Kae stimulates osteogenic differentiation in cultured osteoblasts through estrogen receptor signaling. In addition, evidence suggests that Kae has a positive effect on bone metabolism, since it was observed to promote osteogenesis and to inhibit osteoclast activity, adipocyte formation and autophagy (26-30). From the in vivo assessment of bone microarchitecture, the present study verified that the bone mass parameters (BMD, BV/TV, Tb.N and Tb.Th) were significantly elevated, and the parameters of osteoporosis (SMI and Tb.Sp) reduced in OVX rats treated with Kae compared with OVX group rats. These changes in bone morphology induced by $\mathrm{Kae}$ in OVX rats are similar to those found in previously published research $(26,27)$. However, the concentration of Kae used in the in vivo experiment of the present study was higher than that of previous studies $(26,27)$. The similarity of the results may be associated with differences in delivery time or the vehicle solution used in the present study. Further research is required to effectively assess the effect of different doses of Kae on bone metabolism. Additionally, when BMSCs were isolated from OVX rats and osteogenesis was induced in vitro, 
A

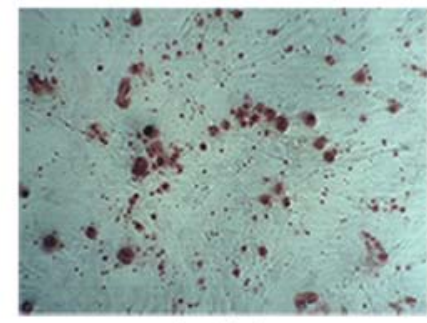

$0 \mu \mathrm{M}$
B

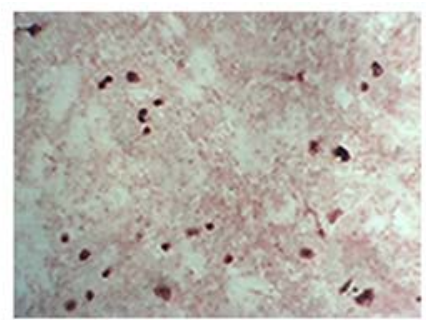

$0.1 \mu \mathrm{M}$
$\mathrm{C}$

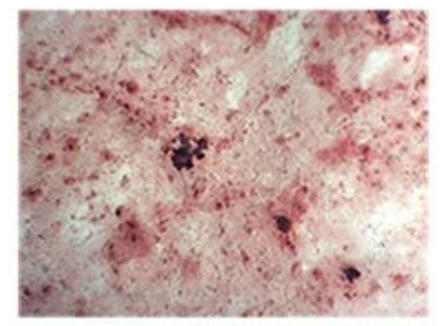

$1 \mu \mathrm{M}$

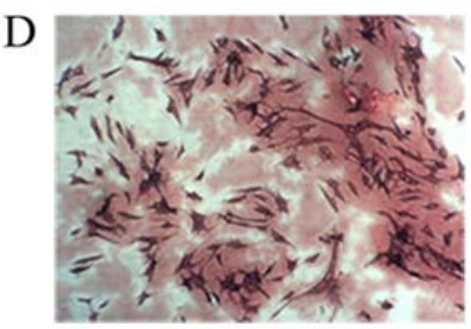

$10 \mu \mathrm{M}$
$\mathrm{E}$

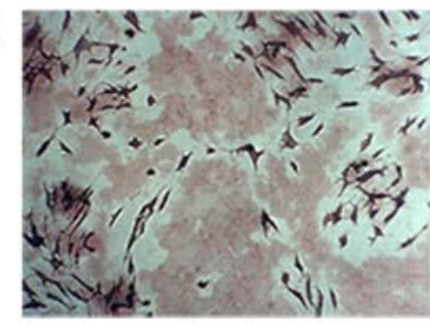

$100 \mu \mathrm{M}$

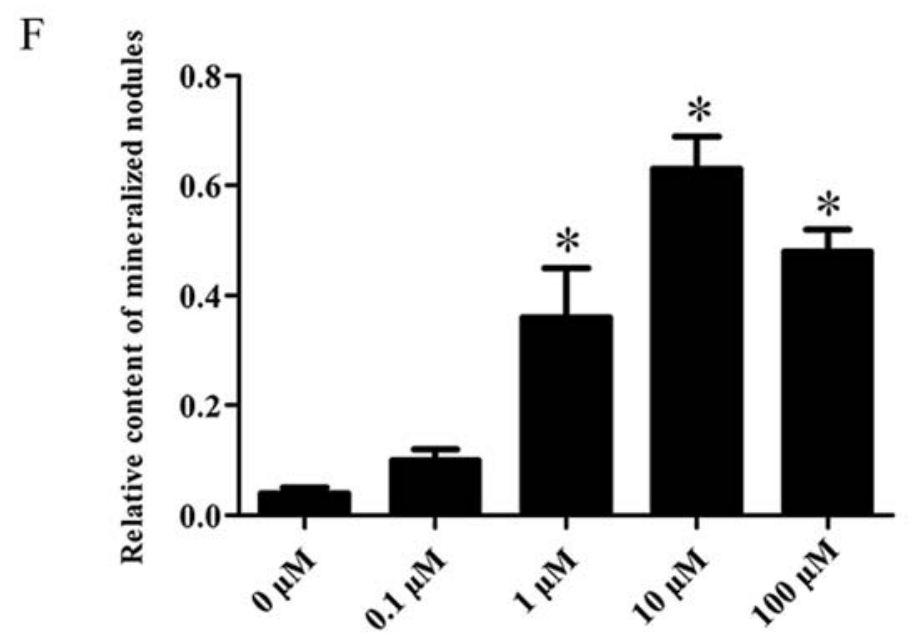

Figure 4. Effects of Kae on BMSC commitment to an osteogenic fate. ARS staining was performed to observe the osteogenic effects of Kae on rat BMSCs $(0-100 \mu \mathrm{M})$. ARS-stained images (magnification, $\mathrm{x} 400$ ) show the effect of the treatment with (A) 0 , (B) 0.1, (C) 1, (D) 10 and (E) $100 \mu \mathrm{M} \mathrm{Kae.} \mathrm{(F)} \mathrm{Bar} \mathrm{graph}$ illustrating the quantitative analysis. Values are presented as the mean \pm SEM of six individual experiments. " $\mathrm{P}<0.05$ vs. $0 \mu$ M. Kae, kaempferol; rBMSCs, rat bone mesenchymal stem cells; ARS, Alizarin Red S.

Kae was found to promote osteogenic differentiation and increase ALP activity, consistent with Kim et al (30), although their data suggest that Kae stimulates osteogenic differentiation through increased expression of autophagy-related factors. In addition, Trivedi et al (26) also reported that treatment with Kae led to a reduction in adipogenic differentiation of BMCs in a Kae + OVX group, verified by a significant reduction in adipocyte number compared with an OVX group using Oil Red O staining. Both osteoblasts and adipocytes share a common precursor (MSCs), and the commitment to either fate is maintained in a dynamic balance under physiological conditions, serving a critical role in the microenvironment of the bone marrow (31-33). However, the imbalance between adipogenesis and osteogenesis has been linked to a number of pathophysiological processes, such as osteoporosis, osteopenia, obesity and aging (31-33). The observations of the present study lend support to these previously published data. Kae principally exerts its osteogenic function via promotion of bone formation, but whether it functions via inhibition of adipogenesis requires further investigation. Together, the in vitro and in vivo experiments suggest that Kae prevented OVX-induced bone loss by promotion of osteoblast function. To determine the molecular mechanism of Kae-dependent osteogenic differentiation, an assessment of the activation of different signaling pathways was performed.

Runx-2, also termed core binding factor $\alpha-1$, activates and initiates the differentiation of BMSCs into osteoblasts and regulates osteoblast maturation (34). Runx-2 is a key transcription factor during osteoblast differentiation and serves a crucial role in bone formation and reconstruction (35). Osterix, a zinc finger-containing transcription factor required for osteoblast differentiation, was first discovered by Nakashima et al in 2002 (36). A study revealed that the silencing of mouse Osterix induces chondrogenesis and chondrocyte differentiation; in addition, bone formation is impaired but Runx 2 expression is unaffected (37). Osterix was not found to be expressed in Runx2-deficient mice, suggesting that it acts downstream of Runx2 during osteoblast differentiation (37). 


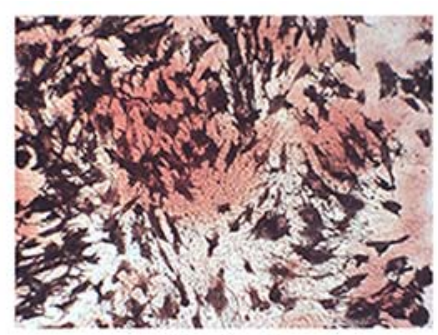

Control

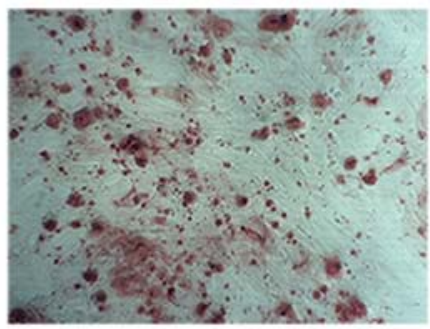

OVX

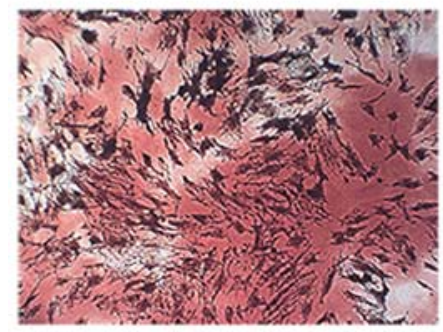

$\mathrm{OVX}+\mathrm{Kae}$

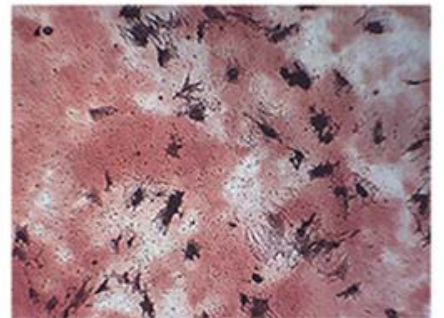

$\mathrm{OVX}+\mathrm{Kae}+\mathrm{Rapa}$

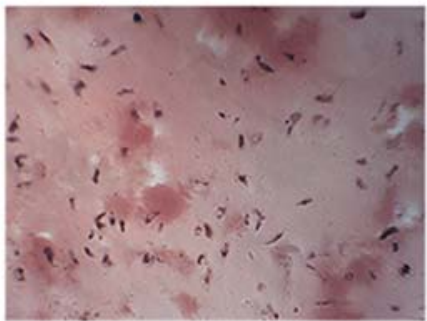

OVX + Rapa

\section{B}

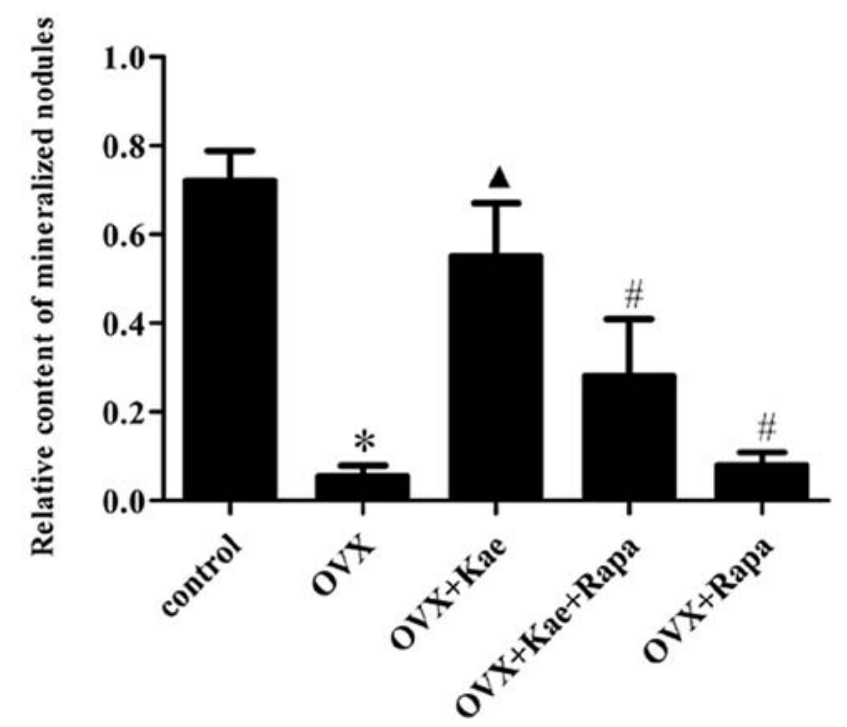

Figure 5. Kae promotes the differentiation of BMSCs into osteoblasts. ARS staining was performed to observe the osteogenic differentiation of cultured BMSCs. (A) ARS-stained images (magnification, $\mathrm{x} 400$ ) and respective (B) quantitative analysis. The data are expressed as the mean \pm SEM ( $=6$ for each group). " $\mathrm{P}<0.05$ vs. control group; ${ }^{\wedge} \mathrm{P}<0.05$ vs. OVX group; ${ }^{\#} \mathrm{P}<0.05$ vs. OVX + Kae group. Kae, kaempferol; BMSCs, bone mesenchymal stem cells; ARS, Alizarin Red S; Rapa, rapamycin; OVX, ovariectomized.

Thus, both Runx2 and Osterix are master transcription factors that promote osteoblast differentiation and bone formation. In the present study, western blot analysis was used to detect the expression levels of Runx 2 and Osterix in BMSCs after the induction of osteogenesis. Kae was found to significantly upregulate Runx 2 and Osterix expression. This suggested that the effect of Kae on osteogenic differentiation is mediated via Runx 2 and Osterix.

mTOR is a member of the phosphatidylinositol 3-kinaserelated kinase family of protein kinases (12). As a core component of intracellular signaling, mTOR regulates cell growth, proliferation and survival, protein synthesis, autophagy and transcription through two functionally and structurally distinct multi-component complexes, mTORC1 and mTORC2 (13). mTORC1 consists of mTOR, regulatory-associated protein of mTOR (Raptor) and G protein $\beta$-unit-like protein (38). mTORC1 promotes the phosphorylation of S6K1, but inhibits the phosphorylation of 4E/BP1 $(39,40)$. Activation of mTORC1 improves growth factor metabolism, cell growth and proliferation $(39,40)$. mTORC2 consists of mTOR, mTOR associated protein, LST8 homolog, Raptor independent companion of mTOR complex 2 and mSIN1, phosphorylating and activating Akt (phosphorylation site, S473) and glycogen synthase kinase phosphorylation $(41,42)$.

Studies have demonstrated that mTOR signaling has numerous key regulatory functions in various diseases, including cancer (43-46), infectious diseases $(47,48)$, atherosclerosis, and degenerative and autoimmune diseases (49-51), and has been suggested to be a novel therapeutic target for the treatment of these diseases (43-51). Other studies have shown that mTOR signaling is also involved in the regulation of multiple aspects of skeletal development (52-54). However, controversy remains 


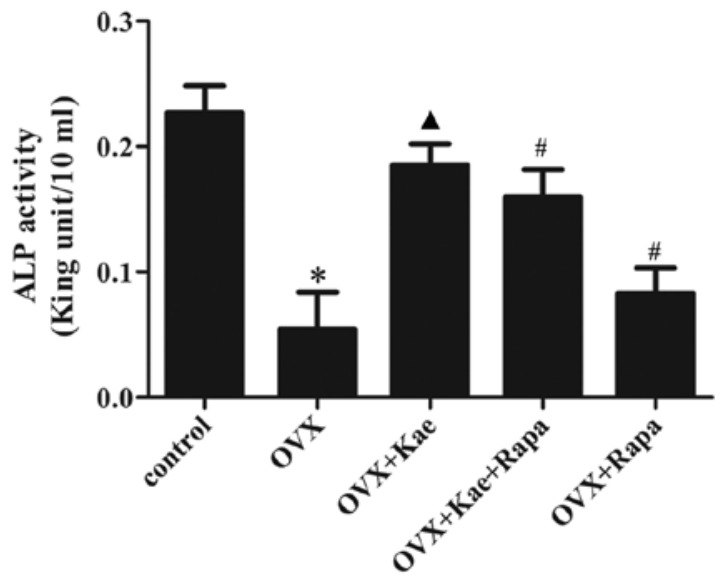

Figure 6. Kae increases the level of ALP in BMSCs. The data are expressed as the mean $\pm \mathrm{SEM}$ ( $\mathrm{n}=6$ for each group). ${ }^{*} \mathrm{P}<0.05$ vs. control group; ${ }^{\wedge} \mathrm{P}<0.05$ vs. OVX group; ${ }^{\#} \mathrm{P}<0.05$ vs. OVX + Kae group. Kae, kaempferol; BMSCs, bone mesenchymal stem cells; ALP, alkaline phosphatase; Rapa, rapamycin; OVX, ovariectomized.

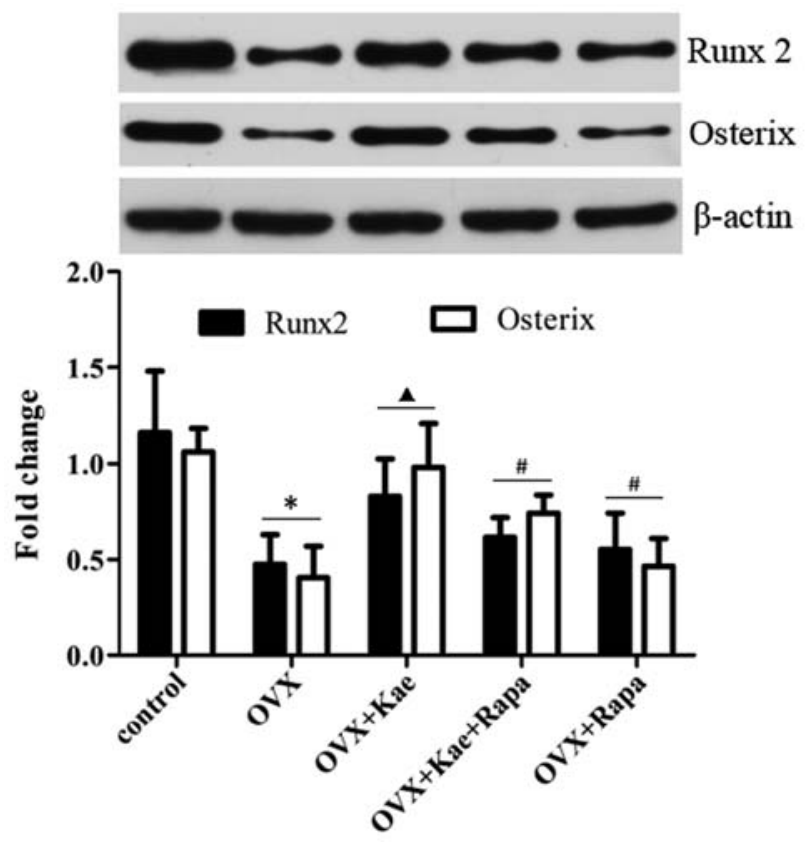

Figure 7. Kae upregulates the expression of Runx 2 and Osterix. Western blot analysis was used for the assessment of Runx2 and Osterix expression, and $\beta$-actin was used as the internal control. Pretreatment with Kae induced increased expression of Runx 2 and Osterix in BMSCs, which was antagonized by Rapa. The data are expressed as the mean \pm SEM ( $n=6$ for each group). ${ }^{*} \mathrm{P}<0.05$ vs. control group; ${ }^{\mathbf{A}} \mathrm{P}<0.05$ vs. OVX group; ${ }^{*} \mathrm{P}<0.05$ vs. $\mathrm{OVX}+\mathrm{Kae}$ group. Kae, kaempferol; BMSCs, bone mesenchymal stem cells; Runx 2, Runt-related transcription factor; Rapa, rapamycin; OVX, ovariectomized.

as to whether mTOR promotes $(17,18,55,56)$ or inhibits $(56-59)$ osteogenic differentiation. A recent study indicated that knockdown of DEP domain containing MTOR interacting protein (Deptor) promotes BMSC osteogenesis both in vitro and in vivo, with higher expression of Deptor contributing to the progression of osteoporosis (56). It is well known that overexpression of Deptor downregulates the activity of mTORC1 and $\mathrm{mTORC} 2$, indicating that mTOR may play a crucial role during osteogenesis. Similarly, a previous study demonstrated
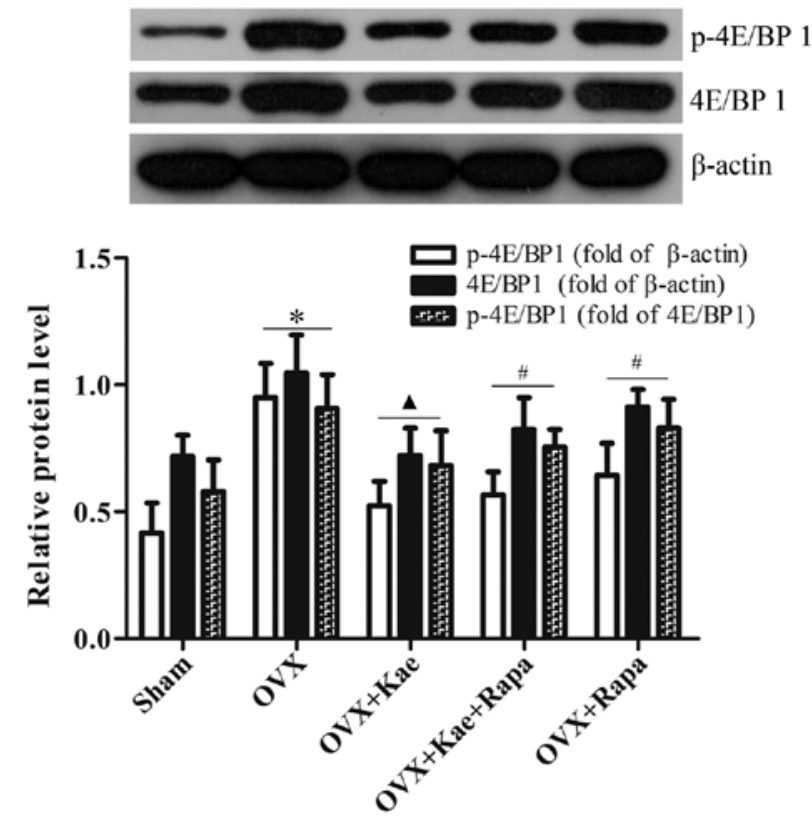

Figure 8. Kae decreases the phosphorylation of 4E/BP1. Relative levels of 4E/BP1 and p-4E/BP1 were detected by western blotting. Treatment with Kae decreased the levels of p-4E/BP1, which was antagonized by Rapa. The data are expressed as the mean \pm SEM ( $n=6$ for each group). ${ }^{*} \mathrm{P}<0.05$ vs. respective sham group; ${ }^{\wedge} \mathrm{P}<0.05$ vs. respective $\mathrm{OVX}$ group; ${ }^{\#} \mathrm{P}<0.05$ vs. respective OVX + Kae group. Kae, kaempferol; Rapa, rapamycin; BMSCs, bone mesenchymal stem cells; 4E/BP1, eukaryotic translation initiation factor 4E-binding protein 1; p, phosphorylated; OVX, ovariectomized.

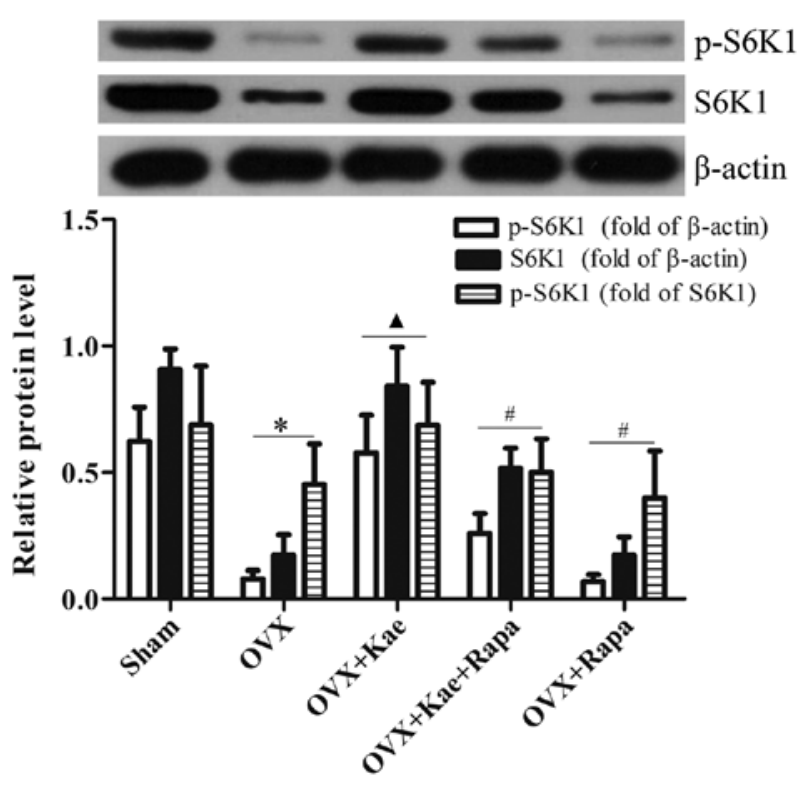

Figure 9. Kae increases the phosphorylation of S6K1. Protein levels of S6K1 and p-S6K1 were detected by western blotting. Treatment with Kae increased the levels of p-S6K1, an effect that was antagonized by Rapa. The data are expressed as the mean \pm SEM ( $\mathrm{n}=6$ for each group). ${ }^{*} \mathrm{P}<0.05$ vs. respective sham group; ${ }^{\wedge} \mathrm{P}<0.05$ vs. respective $\mathrm{OVX}$ group; ${ }^{\#} \mathrm{P}<0.05$ vs. respective OVX + Kae group. Kae, kaempferol; Rapa, rapamycin; BMSCs, bone mesenchymal stem cells; S6K1, ribosomal protein S6 kinase B1; p, phosphorylated; OVX, ovariectomized.

that treatment with Rapa attenuated the expression of ALP, downregulated the expression and phosphorylation of S6K1 and inhibited osteoblastic differentiation in vascular smooth 
muscle cells (VSMCs), suggesting that mTOR participates in the osteoblastic differentiation of VSMCs (18). In the present study, Kae was found to increase the expression of important downstream regulatory factors in the mTOR pathway, which also indicates that mTOR signaling may be involved in osteogenesis. However, a number of researchers have published contrary results. For instance, Dai et al $(59,60)$ indicated that deletion of Raptor (an indispensable component of mTORC1) in osteoclasts leads to increased bone volume and decreased bone resorption. In other words, mTORC1 is critical for osteoclast formation. Raptor deficiency in osteoclasts impairs bone resorption without affecting bone formation, ultimately leading to increased bone mass in mice. On the other hand, $\mathrm{Xu}$ et al (58) reported that the activation of mTORC1 in $\mathrm{B}$ lymphocytes promotes osteoclast formation and causes osteoporosis in mice by promoting TNF superfamily member 11 expression with negative regulation of Akt and $\beta$-catenin. A separate study by Wang et al (61) also indicated that suppression of the mTOR signaling pathway by rapamycin was able to promote BMSC differentiation into osteoblasts in the context of degenerative scoliosis. These conflicting results may be attributed to different methodologies (different cell lines, drug concentrations or experimental design), and underline the requirement for further research. Thus, for further validation, BMSCs treated with Rapa, a specific inhibitor of mTOR, exhibited a marked decline in osteogenic activity and decreased Runx 2 and Osterix expression. This treatment also reversed the positive effects of Kae exposure. Similarly, a number of studies have revealed that Kae can exert cytoprotective effects via regulation of mTOR signaling in different cell lines (62-64). Taken together, these data support the hypothesis that the mTOR-Runx2/Osterix signaling axis is associated with the osteogenic effect of Kae in OVX rats and respective BMSCs.

In summary, the present study demonstrated that Kae has a positive effect on bone formation and osteogenesis in OVX rats, which is possibly exerted via mTOR-Runx2/Osterix signaling. Since Kae naturally occurs in a variety of foods, it is likely that it may exert beneficial effects as a food supplement by improving postmenopausal osteoporosis. Further large scale randomized in vivo trials are now required to confirm the potential of Kae in the treating osteoporosis.

\section{Acknowledgements}

Not applicable.

\section{Funding}

The present study was supported by the Traditional Chinese Medicine Project of the Health and Family Planning Commission of Jiangxi Province, China (grant no. 2017A274) and the Science and Technology Program of Health and Family Planning Commission of Jiangxi Province, China (grant no. 20185232).

\section{Availability of data and materials}

The datasets used and/or analyzed during the present study are available from the corresponding author on reasonable request.

\section{Authors' contributions}

JL conceived and designed the present study and revised the manuscript for important intellectual content. JZ was involved in all experiments and was a major contributor in writing the manuscript. JW modified the study design and designed the structure of the article. YL performed the experiments, analyzed the data and wrote the manuscript. JM and XL completed the data analysis. BX and ZY performed the cell culture, ARS staining, ALP activity assay, western blotting. All authors read and approved the final manuscript.

\section{Ethics approval and consent to participate}

Experiments were performed according to the Guide for the Care and Use of Laboratory (National Institutes of Health), and were approved by the Ethics Committee of Nanchang University (no. 2017-0122).

\section{Patient consent for publication}

Not applicable.

\section{Competing interests}

The authors declare that they have no competing interests.

\section{References}

1. Cosman F, de Beur SJ, LeBoff MS, Lewiecki EM, Tanner B, Randall S and Lindsay R; National Osteoporosis Foundation: Clinician's guide to prevention and treatment of osteoporosis. Osteoporos Int 25: 2359-2381, 2014.

2. Das S and Crockett JC: Osteoporosis-a current view of pharmacological prevention and treatment. Drug Des Devel Ther 7: 435-448, 2013.

3. Rizzoli R, Reginster JY, Boonen S, Bréart G, Diez-Perez A, Felsenberg D, Kaufman JM, Kanis JA and Cooper C: Adverse reactions and drug-drug interactions in the management of women with postmenopausal osteoporosis. Calcif Tissue Int 89: 91-104, 2011.

4. Adler RA, El-Hajj Fuleihan G, Bauer DC, Camacho PM, Clarke BL, Clines GA, Compston JE, Drake MT, Edwards BJ, Favus MJ, et al: Managing osteoporosis in patients on long-term bisphosphonate treatment: Report of a task force of the American society for bone and mineral research. J Bone Miner Res 31: 16-35, 2016.

5. Drieling RL, LaCroix AZ, Beresford SAA, Boudreau DM, Kooperberg C, Chlebowski RT, Ko MG and Heckbert SR: Long-Term oral bisphosphonate therapy and fractures in older women: The Women's health initiative. J Am Geriatr Soc 65: 1924-1931, 2017.

6. Huang L, Yagura T and Chen S: Sedative activity of hexane extract of Keampferia galangal $L$. and its active compounds. J Ethnopharmacol 120: 123-125, 2008.

7. Devi KP, Malar DS, Nabavi SF, Sureda A, Xiao J, Nabavi SM and Daglia M: Kaempferol and inflammation: From chemistry to medicine. Pharmacol Res 99: 1-10, 2015.

8. Qin Y, Cui W, Yang X and Tong B: Kaempferol inhibits the growth and metastasis of cholangiocarcinoma in vitro and in vivo. Acta Biochim Biophys Sin (Shanghai) 48: 238-245, 2016.

9. Suchal K, Malik S, Khan SI, Malhotra RK, Goyal S, Bhatia J, Ojha S and Arya D: Molecular pathways involved in the amelioration of myocardial injury in diabetic rats by kaempferol. Int J Mol Sci 18: pii: E1001, 2017.

10. An M and Kim M: Protective effects of kaempferol against cardiac sinus node dysfunction via CaMKII deoxidization. Anat Cell Biol 48: 235-243, 2015.

11. Mühlbauer RC and Li F: Effect of vegetables on bone metabolism. Nature 401: 343-344, 1999. 
12. Mitra A,Luna JI, Marusina AI, Merleev A, Kundu-Raychaudhuri S, Fiorentino D, Raychaudhuri SP and Maverakis E: Dual mTOR inhibition is required to prevent TGF- $\beta$-mediated fibrosis: Implications for scleroderma. J Invest Dermatol 135: 2873-2876, 2015.

13. Conciatori F, Ciuffreda L, Bazzichetto C, Falcone I, Pilotto S, Bria E, Cognetti F and Milella M: mTOR Cross-talk in cancer and potential for combination therapy. Cancers (Basel) 10: pii: E23, 2018

14. Karner CM, Lee SY and Long F: Bmp induces osteoblast differentiation through both Smad4 and mTORC1 signaling. Mol Cell Biol 37: pii: e00253-16, 2017.

15. Fitter S, Matthews MP, Martin SK, Xie J, Ooi SS, Walkley CR, Codrington JD, Ruegg MA, Hall MN, Proud CG, et al: mTORC1 Plays an important role in skeletal development by controlling preosteoblast differentiation. Mol Cell Biol 37: pii: e00668-16, 2017.

16. Liu DM, Zhao L, Liu TT, Jiao PL, Zhao DD, Shih MS, Tao B, Sun LH, Zhao HY and Liu JM: Rictor/mTORC2 loss in osteoblasts impairs bone mass and strength. Bone 90: 50-58, 2016.

17. Chen J, Tu X, Esen E, Joeng KS, Lin C, Arbeit JM, Rüegg MA, Hall MN, Ma L and Long F: WNT7B promotes bone formation in part through mTORC1. PLoS Genet 10: e1004145, 2014.

18. Zhan JK, Wang YJ, Wang Y, Wang S, Tan P, Huang W and Liu YS: The mammalian target of rapamycin signalling pathway is involved in osteoblastic differentiation of vascular smooth muscle cells. Can J Cardiol 30: 568-575, 2014.

19. Min J, Yuan Z, Zhang Q, Lin S, Wang K and Luo J: Analysis of anti-osteoporosis function of chlorogenic acid by gene microarray profiling in ovariectomy rat model. Biosci Rep 38 : pii: BSR20180775, 2018.

20. Kilkenny C, Browne WJ, Cuthill IC, Emerson M and Altman DG: Improving bioscience research reporting: The ARRIVE guidelines for reporting animal research. PLoS Biol 8: e1000412,2010.

21. Jiang Y, Jahagirdar BN, Reinhardt RL, Schwartz RE, Keene CD, Ortiz-Gonzalez XR, Reyes M, Lenvik T, Lund T, Blackstad M, et al: Pluripotency of mesenchymal stem cells derived from adult marrow. Nature 418: 41-49, 2002.

22. Riggs BL, Khosla S and Melton LJ III: Sex steroids and the construction and conservation of the adult skeleton. Endocr Rev 23: 279-302, 2002.

23. Chien KR and Karsenty G: Longevity and lineages: Toward the integrative biology of degenerative diseases in heart, muscle, and bone. Cell 120: 533-544, 2005.

24. Hegarty VM, May HM and Khaw KT: Tea drinking and bone mineral density in older women. Am J Clin Nutr 71: 1003-1007, 2000.

25. Guo AJ, Choi RC, Zheng KY, Chen VP, Dong TT, Wang ZT, Vollmer G, Lau DT and Tsim KW: Kaempferol as a flavonoid induces osteoblastic differentiation via estrogen receptor signaling. Chin Med 7: 10, 2012.

26. Trivedi R, Kumar S, Kumar A, Siddiqui JA, Swarnkar G, Gupta V, Kendurker A, Dwivedi AK, Romero JR and Chattopadhyay N: Kaempferol has osteogenic effect in ovariectomized adult Sprague-Dawley rats. Mol Cell Endocrinol 289: 85-93, 2008.

27. Nowak B, Matuszewska A, Nikodem A, Filipiak J, Landwójtowicz M, Sadanowicz E, Jędrzejuk D, Rzeszutko M, Zduniak K, Piasecki T, et al: Oral administration of kaempferol inhibits bone loss in rat model of ovariectomy-induced osteopenia. Pharmacol Rep 69: 1113-1119, 2017.

28. Kim CJ, Shin SH, Kim BJ, Kim CH, Kim JH, Kang HM, Park BS and Kim IR: The effects of Kaempferol-inhibited autophagy on osteoclast formation. Int J Mol Sci 19: pii: E125, 2018.

29. Adhikary S, Choudhary D, Ahmad N, Karvande A, Kumar A, Banala VT, Mishra PR and Trivedi R: Dietary flavonoid kaempferol inhibits glucocorticoid-induced bone loss by promoting osteoblast survival. Nutrition 53: 64-76, 2018.

30. Kim IR, Kim SE, Baek HS, Kim BJ, Kim CH, Chung IK, Park BS and Shin SH: The role of kaempferol-induced autophagy on differentiation and mineralization of osteoblastic MC3T3-E1 cells. BMC Complement Altern Med 16: 333, 2016.

31. Chen Q, Shou P, Zheng C, Jiang M, Cao G, Yang Q, Cao J, Xie N, Velletri T, Zhang X, et al: Fate decision of mesenchymal stem cells: Adipocytes or osteoblasts? Cell Death Differ 23 $1128-1139,2016$

32. Eriksen EF: Cellular mechanisms of bone remodeling. Rev Endocr Metab Disord 11: 219-227, 2010.

33. Zuk PA, Zhu M, Ashjian P, De Ugarte DA, Huang JI, Mizuno H, Alfonso ZC, Fraser JK, Benhaim P and Hedrick MH: Human adipose tissue is a source of multipotent stem cells. Mol Biol Cell 13: 4279-4295, 2002.
34. Lucero CM, Vega OA, Osorio MM, Tapia JC, Antonelli M, Stein GS, van Wijnen AJ and Galindo MA: The cancer-related transcription factor Runx 2 modulates cell proliferation in human osteosarcoma cell lines. J Cell Physiol 228: 714-723, 2013.

35. Wysokinski D, Pawlowska E and Blasiak J: RUNX2: A master bone growth regulator that may be involved in the DNA damage response. DNA Cell Biol 34: 305-315, 2015.

36. Nakashima K, Zhou X, Kunkel G, Zhang Z, Deng JM, Behringer RR and de Crombrugghe $B$ : The novel zinc finger-containing transcription factor osterix is required for osteoblast differentiation and bone formation. Cell 108: 17-29, 2002.

37. Nishimura R, Wakabayashi M, Hata K, Matsubara T, Honma S, Wakisaka S, Kiyonari H, Shioi G, Yamaguchi A, Tsumaki N, et al: Osterix regulates calcification and degradation of chondrogenic matrices through matrix metalloproteinase 13 (MMP13) expression in association with transcription factor Runx 2 during endochondral ossification. J Biol Chem 287: 33179-33190, 2012.

38. Zoncu R, Efeyan A and Sabatini DM: mTOR: From growth signal integration to cancer, diabetes and ageing. Nat Rev Mol Cell Biol 12: 21-35, 2011.

39. Howell JJ and Manning BD: mTOR couples cellular nutrient sensing to organismal metabolic homeostasis. Trends Endocrinol Metab 22: 94-102, 2011

40. Dai N, Rapley J, Angel M, Yanik MF, Blower MD and Avruch J: mTOR phosphorylates IMP2 to promote IGF2 mRNA translation by internal ribosomal entry. Genes Dev 25: 1159-1172, 2011.

41. Cybulski N and Hall MN: TOR complex 2: A signaling pathway of its own. Trends Biochem Sci 34: 620-627, 2009.

42. Alessi DR, Pearce LR and García-Martínez JM: New insights into mTOR signaling: MTORC2 and beyond. Sci Signal 2: pe27, 2009.

43. Steelman LS, Martelli AM, Cocco L, Libra M, Nicoletti F, Abrams SL and McCubrey JA: The therapeutic potential of mTOR inhibitors in breast cancer. Br J Clin Pharmacol 82: 1189-1212, 2016.

44. Liu ZG, Tang J, Chen Z, Zhang H, Wang H, Yang J and Zhang H: The novel mTORC1/2 dual inhibitor INK128 enhances radiosensitivity of breast cancer cell line MCF-7. Int J Oncol 49: $1039-1045,2016$

45. Ling S, Song L, Fan N, Feng T, Liu L, Yang X, Wang M, Li Y, Tian Y, Zhao F, et al: Combination of metformin and sorafenib suppresses proliferation and induces autophagy of hepatocellular carcinoma via targeting the mTOR pathway. Int J Oncol 50: 297-309, 2017.

46. Guo H, Xu Y, Wang F, Shen Z, Tuo X, Qian H, Wang H and Wang K: Clinical associations between ASCT2 and p-mTOR in the pathogenesis and prognosis of epithelial ovarian cancer. Oncol Rep 40: 3725-3733, 2018.

47. Nicoletti F, Fagone P, Meroni P, McCubrey J and Bendtzen K: mTOR as a multifunctional therapeutic target in HIV infection. Drug Discov Today 16: 715-721, 2011.

48. Donia M, McCubrey JA, Bendtzen K and Nicoletti F: Potential use of rapamycin in HIV infection. Br J Clin Pharmacol 70: 784-793, 2010

49. Mammana S, Bramanti P, Mazzon E, Cavalli E, Basile MS, Fagone P, Petralia MC, McCubrey JA, Nicoletti F and Mangano K: Preclinical evaluation of the PI3K/Akt/mTOR pathway in animal models of multiple sclerosis. Oncotarget 9: 8263-8277, 2018.

50. Donia M, Mangano K, Amoroso A, Mazzarino MC, Imbesi R, Castrogiovanni P, Coco M, Meroni P and Nicoletti F: Treatment with rapamycin ameliorates clinical and histological signs of protracted relapsing experimental allergic encephalomyelitis in Dark Agouti rats and induces expansion of peripheral CD4+CD25+Foxp3+regulatory T cells.J Autoimmun 33: 135-140, 2009.

51. Oaks Z, Winans T, Huang N, Banki K and Perl A: Activation of the mechanistic target of Rapamycin in SLE: Explosion of evidence in the last five years. Curr Rheumatol Rep 18: 73, 2016

52. Chen J and Long F: mTOR signaling in skeletal development and disease. Bone Res 6: 1, 2018.

53. Wu H, Wu Z, Li P, Cong Q, Chen R, Xu W, Biswas S, Liu H, $\mathrm{Xia} X, \mathrm{Li} \mathrm{S}$, et al: Bone size and quality regulation: Concerted actions of mTOR in mesenchymal stromal cells and osteoclasts. Stem Cell Reports 8: 1600-1616, 2017.

54. Chen C, Akiyama K, Wang D, Xu X, Li B, Moshaverinia A, Brombacher F, Sun L and Shi S: mTOR inhibition rescues osteopenia in mice with systemic sclerosis. J Exp Med 212: 73-91,2015. 
55. Li SF, Tang JJ, Chen J, Zhang P, Wang T, Chen TY, Yan B, Huang B, Wang L, Huang MJ, et al: Regulation of bone formation by baicalein via the mTORC1 pathway. Drug Des Devel Ther 9: 5169-5183, 2015

56. Chen S, Jia L, Zhang S, Zheng Y and Zhou Y: DEPTOR regulates osteogenic differentiation via inhibiting MEG3-mediated activation of BMP4 signaling and is involved in osteoporosis. Stem Cell Res Ther 9: 185, 2018.

57. Martin SK, Fitter S, Bong LF, Drew JJ, Gronthos S, Shepherd PR and Zannettino AC: NVP-BEZ235, a dual pan class I PI3 kinase and $\mathrm{mTOR}$ inhibitor, promotes osteogenic differentiation in human mesenchymal stromal cells. J Bone Miner Res 25: 2126-2137, 2010

58. Xu S, Zhang Y, Liu B, Li K, Huang B, Yan B, Zhang Z, Liang K, $\mathrm{Jia} \mathrm{C}$, Lin $\mathrm{J}$, et al: Activation of mTORC1 in B lymphocytes promotes osteoclast formation via regulation of $\beta$-catenin and RANKL/OPG. J Bone Miner Res 31: 1320-1333, 2016.

59. Dai Q, Han Y, Xie F, Ma X, Xu Z, Liu X, Zou W and Wang J: A RANKL-based osteoclast culture assay of mouse bone marrow to investigate the role of mTORC1 in osteoclast formation. J Vis Exp: Mar 15, 2018 doi: 10.3791/56468.

60. Dai Q, Xie F, Han Y, Ma X, Zhou S, Jiang L, Zou W and Wang J: Inactivation of Regulatory-associated protein of mTOR (Raptor)/mammalian target of rapamycin complex 1 (mTORC1) signaling in osteoclasts increases bone mass by inhibiting osteoclast differentiation in mice. J Biol Chem 292: 196-204, 2017.
61. Wang Y, Yi XD and Li CD: Suppression of mTOR signaling pathway promotes bone marrow mesenchymal stem cells differentiation into osteoblast in degenerative scoliosis: In vivo and in vitro. Mol Biol Rep 44: 129-137, 2017.

62. Che J, Liang B, Zhang Y, Wang Y, Tang J and Shi G: Kaempferol alleviates ox-LDL-induced apoptosis by up-regulation of autophagy via inhibiting PI3K/Akt/mTOR pathway in human endothelial cells. Cardiovasc Pathol 31: 57-62, 2017.

63. Varshney R, Gupta S and Roy P: Cytoprotective effect of kaempferol against palmitic acid-induced pancreatic $\beta$-cell death through modulation of autophagy via AMPK/mTOR signaling pathway. Mol Cell Endocrinol 448: 1-20, 2017.

64. Kim GD: Kaempferol inhibits angiogenesis by suppressing HIF-1 $\alpha$ and VEGFR2 Activation via ERK/p38 MAPK and PI3K/Akt/mTOR signaling pathways in endothelial cells. Prev Nutr Food Sci 22: 320-326, 2017. International (CC BY-NC-ND 4.0) License. 